SLAC-PUB-8112

April 1999

\title{
The SLAC Linac during the PEP-II Era"
}

\author{
F.-J. Decker, R.H. Iverson, H. Smith, M.S. Zelazny, \\ Stanford Linear Accelerator Center, Stanford University, Stanford, CA 94309, USA
}

\begin{abstract}
The $3 \mathrm{~km}$ long linac of the Stanford Linear Accelerator Center (SLAC) has for many years provided low emittance beams for the SLC collider. With the beginning of the operation of the B-Factory, PEP-II, the linac will now serve primarily as an injector for the low (LER) and high energy rings (HER) of PEP-II. The different beams are stabilized by a new regional linac energy management (LEM) program, which keeps the magnet strengths and therefore the deflections constant at each of the four extraction points. They are at 3.1 $\mathrm{GeV}$ (positrons for LER), $9 \mathrm{GeV}$ (electrons for HER), $25 \mathrm{GeV}$ (electrons for the positron production), and $28.5 \mathrm{GeV}$ (electrons for other purposes such as test beams, plasma experiments, and iris destruction tests). Since many components of the accelerator can be configured on each pulse, it is possible to run these beams with different intensities, bunch length, energies, rates, etc. Only the quadrupole magnet strengths are fixed, producing different lattices for different energy beams, which must be matched at extraction from the linac. Since PEP-II injection does not require all of the full $120 \mathrm{~Hz}$ rate, it is possible to run other experiments at the same time. That might include an extension of the SLC run or a high charge, fixed target experiment, which will require some additional pulsed magnets.
\end{abstract}

Contributed to the IEEE Particle Accelerator Conference, PAC99

New York, NY, 29-Mar-1999 to 2-Apr-1999

* Work supported by Department of Energy contract DE-AC03-76SF00515. 


\title{
The SLAC Linac during the PEP-II Era
}

\author{
F.-J. Decker, R.H. Iverson, H. Smith, M.S. Zelazny, \\ Stanford Linear Accelerator Center*, Stanford, CA 94309, USA
}

\begin{abstract}
The $3 \mathrm{~km}$ long linac of the Stanford Linear Accelerator Center (SLAC) has for many years provided low emittance beams for the SLC collider. With the beginning of the operation of the B-Factory, PEP-II, the linac will now serve primarily as an injector for the low (LER) and high energy rings (HER) of PEP-II. The different beams are stabilized by a new regional linac energy management (LEM) program, which keeps the magnet strengths and therefore the deflections constant at each of the four extraction points. They are at $3.1 \mathrm{GeV}$ (positrons for LER), $9 \mathrm{GeV}$ (electrons for HER), $25 \mathrm{GeV}$ (electrons for the positron production), and $28.5 \mathrm{GeV}$ (electrons for other purposes such as test beams, plasma experiments, and iris destruction tests). Since many components of the accelerator can be configured on each pulse, it is possible to run these beams with different intensities, bunch length, energies, rates, etc. Only the quadrupole magnet strengths are fixed, producing different lattices for different energy beams, which must be matched at extraction from the linac. Since PEP-II injection does not require all of the full $120 \mathrm{~Hz}$ rate, it is possible to run other experiments at the same time. That might include an extension of the SLC run or a high charge, fixed target experiment, which will require some additional pulsed magnets.
\end{abstract}

\section{INTRODUCTION}

For the SLC-collider, the SLAC linac ran at $120 \mathrm{~Hz}$ and co-accelerated three beams together: positrons, electrons and a scavenger bunch to the positron target at $2 / 3$ of the linac length. For the PEP-II era the main purpose is to provide a stable fill rate for the low and high energy ring. The linac runs mainly at $30 \mathrm{~Hz}$ till $2 / 3$ of the linac and 60 $\mathrm{Hz}$ in the first 3 sectors till the extraction of the positrons into the by-pass line. So there are the following rates available: $10 \mathrm{~Hz}$ for $\mathrm{e}^{-}, 10 \mathrm{~Hz}$ for $\mathrm{e}^{-}$(scav) and an extra 10 $\mathrm{Hz}$ for an $\mathrm{e}^{-}$-beam for additional experiments (like plasma acceleration or plasma lens in final focus test beam area, FFTB). The positrons are in between this in a $60 \mathrm{~Hz}$ timeslot. On demand this can be changed to $30 \mathrm{~Hz} \mathrm{e}^{-}$to HER, or $30 \mathrm{~Hz} \mathrm{e}^{-}$(scav) and $30 \mathrm{~Hz} \mathrm{e}^{+}$to LER for a three times faster fill rate.

Besides the scheduling issues, the linac has to provide stable and sometimes special beam properties for the different beams.

*Work supported by Department of Energy contract DE-AC0376 SF00515.

\section{NEW LEM}

The linac energy management program (LEM) keeps the magnet focussing lattice stable by accounting for the different klystrons which accelerate the beam. Since each klystron can be on for one beam and off for another, it is impossible to adjust the magnets to the same lattice on a pulse by pulse basis. Different strategies are possible.

\section{1 "Anti-LEM"}

During the SLC-run the PEP-positrons got decelerated in their last part in the linac and got after each quadrupole adjustment by LEM a big kick in the last quadrupole where they are off-axis. This needed corrector changes (antiLEM) and re-matching in some degree.

\subsection{Regional LEM}

To avoid these frequent adjustments, a regional LEM was introduced, which keeps the end energy and magnet strengths for each of the four beams constant $\left(\mathrm{e}^{+}: 3.1 \mathrm{GeV}\right.$, $\mathrm{e}^{-}: 9 \mathrm{GeV}, \mathrm{e}^{-}$(scav): $\left.25 \mathrm{GeV}, \mathrm{e}^{-}(\mathrm{FFTB}): 28.5 \mathrm{GeV}\right)$. This is only perfect when each beam has the same acceleration. But since this keeps the lowest energy beam constant, a higher energy, mismatched beam will see a weaker lattice, which is not too bad.

\subsection{Additional Matching}

In principle this mismatched beam could be re-matched after the lower energy beam got extracted from the linac. This was never tried and has the potential of disturbing other careful adjusted beam parameters like wakefield bumps which cancel unknown errors at a special betatron phases.

\subsection{Identical Energy Profiles}

This leaves only the solution of identical energy profiles. But since the FFTB beam needs a big initial energy spread for transverse stability, which is accomplished by a $20^{\circ}$ phase offset (BNS-damping), the energy would be different again, but the following trick helps. By timing the klystrons (which use SLED) for the positrons pulse $240 \mathrm{~ns}$ late, so that the accelerator structure is not completely filled, the $\mathrm{e}^{+}$-energy on the rf-crest is adjusted to be identical to the FFTB beam, which is $20^{\circ}$ off crest (see Fig. 1). 

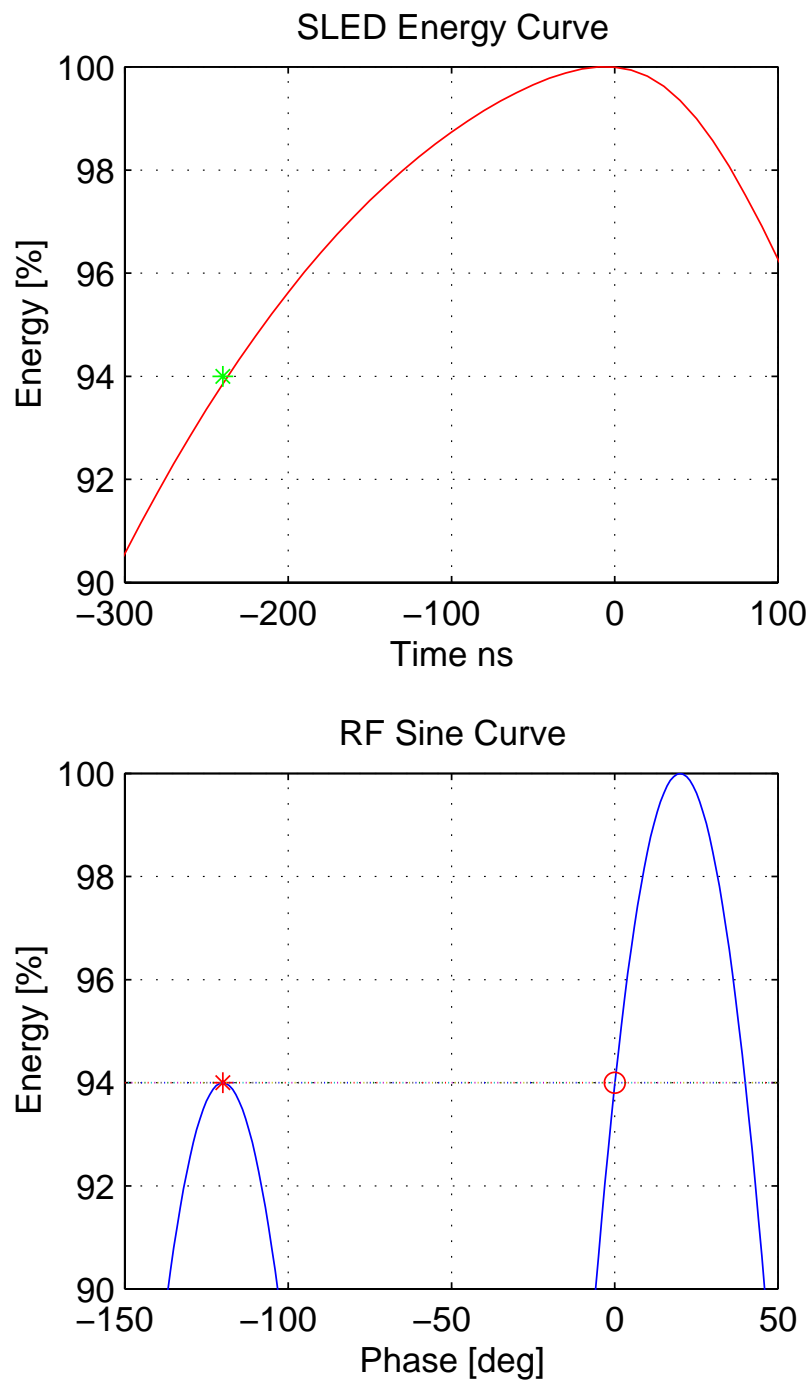

Fig. 1: Equal beam energies.

Injecting the positrons $240 \mathrm{~ns}$ early on crest (left) gives the same energy like being $20^{\circ}$ off crest but on the peak of the (SLEDded) klystron fill curve (right).

\section{SENSITIVITY TO BEAM CHANGES}

Since the PEP-II rings are not very sensitive to changing beam emittances or offsets, the tuning is much relaxed, just the availability for filling counts. This might change when background issues get more important with the BaBar detector in place. On the other hand the FFTB beam for the plasma experiments has to be maintained to much better accuracy. For instance, when the linac steering feedbacks were keeping the $\mathrm{e}^{-}$(scav) "stable", the emittance for the FFTB beam changed easily by a factor of three. By switching the feedback to the FFTB beam, this beam has stabilised, but also helped to keep the other beams in a smaller range of variations.

\section{POSITRON ENERGY SPREAD}

Since the positrons have with $3.1 \mathrm{GeV}$ the smallest energy, their energy spread is still dominated by the energy spread introduced by the compression in the ring-to-linac section (RTL). With a damping ring bunch length of $6 \mathrm{~mm}$ and an energy spread of $0.07 \%$ at $1.19 \mathrm{GeV}$, the following energy spreads are possible (see Tab. 1). A total compression on a linear slope is assumed, and wakefields and the rf sine curvature in the linac are ignored.

Table 1: Possible Energy Spreads.

\begin{tabular}{|c|c|c|c|}
\hline $\begin{array}{c}\text { Com- } \\
\text { pressor }\end{array}$ & $\Delta \boldsymbol{E}$ & $\sigma_{\boldsymbol{z}}$ & $\begin{array}{c}\Delta \boldsymbol{E} / \boldsymbol{E} \\
\text { at 3.1 GeV }\end{array}$ \\
\hline $30 \mathrm{MeV}$ & $10.7 \mathrm{MeV}$ & $0.47 \mathrm{~mm}$ & $0.34 \%$ \\
\hline $25 \mathrm{MeV}$ & $8.9 \mathrm{MeV}$ & $0.56 \mathrm{~mm}$ & $0.28 \%$ \\
\hline $15 \mathrm{MeV}$ & $5.3 \mathrm{MeV}$ & $0.94 \mathrm{~mm}$ & $0.17 \%$ \\
\hline $12 \mathrm{MeV}$ & $4.3 \mathrm{MeV}$ & $1.18 \mathrm{~mm}$ & $0.14 \%$ \\
\hline $10 \mathrm{MeV}$ & $3.6 \mathrm{MeV}$ & $1.41 \mathrm{~mm}$ & $0.11 \%$ \\
\hline
\end{tabular}

This table seems to be in discrepancy with the data where at $27 \mathrm{MeV}$ a $0.15 \%$ energy spread is achieved (see below).

\subsection{Longitudinal Emittance}

The longitudinal emittance is given by the damping ring rf. The maximum reduction is about $10 \%$, by raising the rf amplitude by $20 \%$.

\subsection{Longitudinal Phase Space}

The longitudinal phase space can be adjusted by lowering the bunch length and raising the energy spread, which is mainly done in the compression region. Three different corrections come to mind to change this adjustment.

- Pre-compression in the damping ring: This will lower the bunch length and therefore the non-linear part of the compression rf.

- Changing $R_{56}$ in the compression region (which is the basic assumption of Table 1): This will require changes of up to a factor of three in magnet strength, which might be not easily feasible.

- Correlated energy spread cancellation: By undercompressing the beam, the bunch length will be longer and there will be an energy-length correlation. This correlation can be taken out later in the linac by accelerating the beam at the right amount off crest. This will reduce the energy spread, giving an explanation why at $27 \mathrm{MeV}$ the energy spread can be as low as $0.15 \%$ in the experiment.

\subsection{Simulations}

To compare the analytic result with reality, the longitudinal phase space changes were simulated taking into account the linac rf and wakefields. Fig. 2 shows the 1-, 2and 3-sigma contour lines after the compression (RTL) 

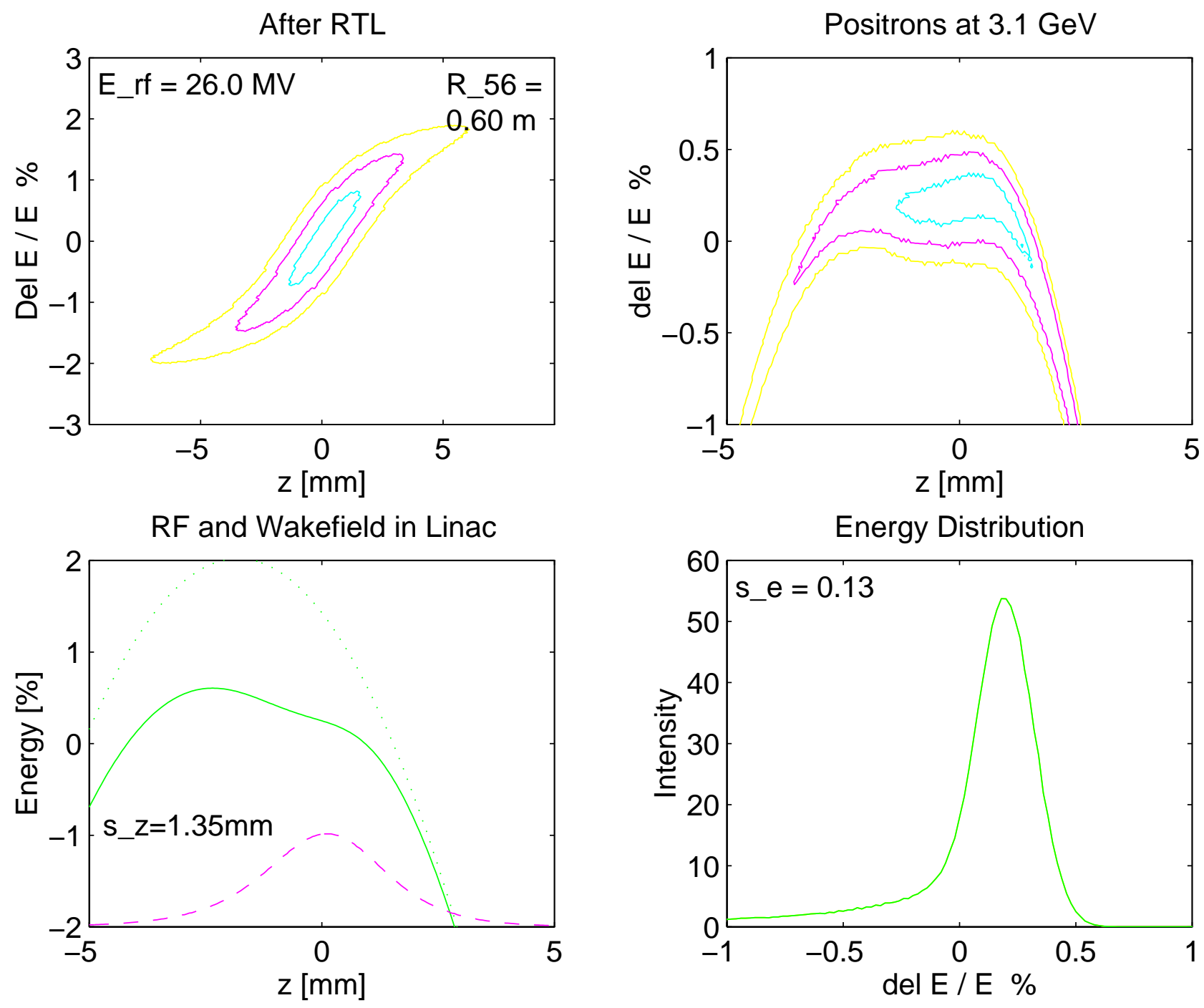

Fig. 2: Low positron energy spread.

By cancelling the correlated energy spread after compression in the RTL with the opposite correlation of the linac rf (including wakefields), a $0.13 \%$ energy spread can be achieved.

and at the $3.1 \mathrm{GeV}$ energy point in the linac. By compensating the $E$-z-slope of the under-compressed beam with the slope created by the rf in the linac (and the wakefield for $2 \cdot 10^{10}$ particles), a fitted energy spread of $0.13 \%$ can be achieved (plus low energy tail). The longitudinal wakefields help to flatten the curve, which allows a longer bunch length, making a better correlation compensation possible.

\section{DIFFERENT BEAM CHARGES}

At $2 \cdot 10^{10}$ particles per bunch the beam loading within the bunch reduces the energy by nearly $2 \%$ (compare Fig. 2). This implies that a very low charge $\left(2 \cdot 10^{9}\right)$ for topping of

and equalising the PEP-II bunches in the ring, will require a different energy and phase in the linac. This is also true for $4 \cdot 10^{10}$ FFTB beams or a fixed target experiment, running together with PEP-II.

\section{SUMMARY}

PEP-II requires beams with different property, filling or topping of the rings and other experiments together with PEP-II. This can be solved mainly by pulsed devices or needs a compromise in the set-up. The positron energy spread is an example, where a careful cancellation can be used to reduce the energy spread by a factor of two.

\section{REFERENCES}

[1] V.E. Balakin, A.V. Novokhatski, V.P. Smirnov, VLEPP, Transverse Beam Dynamics, $12^{\text {th }}$ Int. Conf. On High Energy Accel., FNAL (1983) 119.

[2] D. Farkas, H. Hoag, G. Loew, P. Wilson, SLED: A Method of Doubling SLAC's Energy, SLAC-PUB 1453, June 1974. 